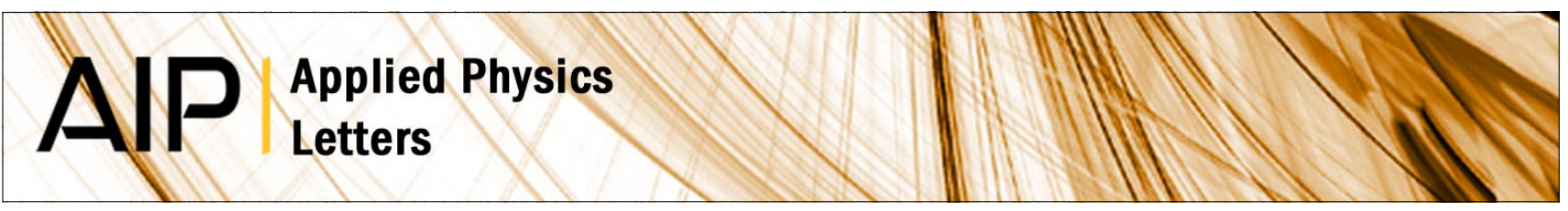

\title{
Quantum well lasers tunable by long wavelength radiation
}

E. Gerck and L. C. M. Miranda

Citation: Appl. Phys. Lett. 44, 837 (1984); doi: 10.1063/1.94942

View online: http://dx.doi.org/10.1063/1.94942

View Table of Contents: http://apl.aip.org/resource/1/APPLAB/v44/i9

Published by the American Institute of Physics.

Additional information on Appl. Phys. Lett.

Journal Homepage: http://apl.aip.org/

Journal Information: http://apl.aip.org/about/about_the_journal

Top downloads: http://apl.aip.org/features/most_downloaded

Information for Authors: http://apl.aip.org/authors

\section{ADVERTISEMENT}

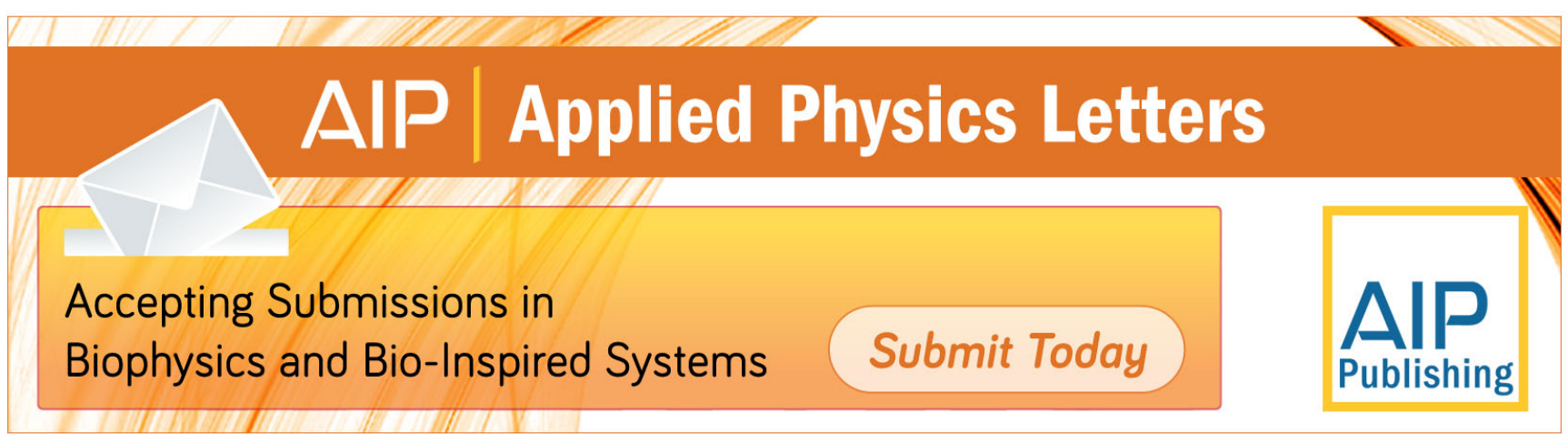




\title{
Quantum well lasers tunable by long wavelength radiation
}

\author{
E. Gerck and L. C. M. Miranda \\ Instituto de Estudos Avançados, Centro Técnico Aeroespacial, 12200-S. J. dos Campos-SP, Brazil
}

(Received 13 September 1983; accepted for publication 14 February 1984)

It is suggested that the lasing frequency of GaAs-GaAlAs quantum well lasers can be tuned when the quantum well structure is irradiated with a pulsed low-energy long-wavelength laser. This new tuning mechanism is based on the decrease of the electron binding energy, in the well, with increasing intensity of the external laser field. Typically, $0.60 \mathrm{~mJ}$ of $10.6-\mu \mathrm{m}$ radiation in a $1-\mathrm{ns}$ pulse may blue-shift the quantum well laser frequency by approximately $20 \mathrm{~nm}$.

PACS numbers: 42.55.Px, 68.55. $+\mathrm{b}$

In recent years GaAs-GaAlAs quantum well lasers ${ }^{1-5}$ (QWL) have attracted the attention of a considerable number of authors. These novel diode structures are undoubtedly very promising as they exhibit the lowest threshold current density $\left(\sim 300 \mathrm{~A} / \mathrm{cm}^{2}\right)$ for lasing to date. For a review on this subject we refer to the work of Holonyak et al. ${ }^{3}$ The well widths in these heterostructures can vary from 75 to $100 \AA$ and their depths are typically of the order of $250 \mathrm{meV}$ for the electrons. In Fig. 1 we show the schematic energy-band diagram associated with the graded-barrier GaAs-GaAlAs quantum well laser of Ref. 5. This laser has a $75-\AA$-wide quantum well with a depth of $270 \mathrm{meV}$, a threshold current density of only $330 \mathrm{~A} / \mathrm{cm}^{2}$, and its emission wavelength occurs at $8440 \AA$ corresponding to the transition energy between electrons in the ground state $(n=1)$ of the potential well and heavy holes.

In this letter we show that the QWL lasing frequency can be tuned when the quantum well structure is irradiated by a pulsed low-energy nonresonant laser. To this end, we consider the simple model in which one electron is confined in a single one-dimensional quantum well of width $a_{0}$ and depth $V_{0}$. The electron is then subjected to the electromagnetic field of the external laser, and the question we want to answer is how the bound states of the confined electron are affected by the additional driving field. The driving laser beam is described by a classical plane electromagnetic wave of frequency $\omega$, linearly polarized along the axis of the well ( $x$ axis), and propagating parallel to the lasing direction of the heterostructure ( $z$ axis). We also assume that this laser beam is nonresonant with the QWL system and that the photon energy of the driving laser is smaller than the field-free binding energy. Under these conditions, the electron motion is thus described by the solution to the Schrödinger equation

$$
i \hbar \dot{\psi}(x, t)=\left[\frac{1}{2 m}\left(\hat{p}+\frac{e}{c} A(t)\right)^{2}+V(x)\right] \psi(x, t),
$$

where $\hat{p}=(\hbar / i)(\partial / \partial x), A(t)=A \sin \omega t$ is the vector potential for the linearly polarized driving laser field in the dipole approximation, and $V(x)$ is the potential well.

To solve Eq. (1) we perform the unitary transformation ${ }^{6}$

$$
\psi=e^{i \delta(t \mid \hat{p} / \hbar} e^{i \eta|t| / \hbar} \phi,
$$

where

$$
\delta(t)=-\frac{e}{m c} \int^{t} d t^{\prime} A\left(t^{\prime}\right)=a \cos \omega t ; \quad a=\frac{e A}{m c \omega}
$$

$$
\eta(t)=-\frac{e^{2}}{2 m c^{2}} \int^{t} d t^{\prime} A^{2}\left(t^{\prime}\right)
$$

We find

$$
i \hbar \dot{\phi}(x, t)=\left\{\hat{p}^{2} / 2 m+V[x-\delta(t)]\right\} \phi(x, t) .
$$

Equation (4) shows that in the presence of a laser field the electron motion may be alternatively described by the Schrödinger equation for an electron moving in a time-dependent well which oscillates with (laser) frequency $\omega$ and amplitude $a=e A / m c \omega$. The potential $V[x-\delta(t)]$ will be called the laser-dressed potential.

Equation (4) has no exact analytical solutions but can be approximately solved in two different ranges of frequencies $\omega$. First, if the period of oscillation of the laser field is much larger than the transit time $\tau$ of the electron in the well $\left(\tau=2 \mathrm{a}_{0} /\langle v\rangle\right.$, where $\langle v\rangle$ is the average speed of the electron in the well ground state), then the electron can reach a steady state in a time $\Delta t \gg \tau$ but still short enough so that $V(x-\delta(t))$ $\simeq V[x-\delta(t+\Delta t)]$. That is, for $\omega \tau \ll 1$, the electron is too fast to see the potential well distortion induced by the laser field. Accordingly, in this low-frequency (or adiabatic) limit one may ignore the laser dressing of the potential well, so that the solutions of Eq. (4) have the same eigenvalues as the laser-free system and no tuning effect is obtained. Physically, the regime of a slowly moving well is characterized by the electron motion being dominated by the undistorted potential. In contrast, in the high-frequency limit, where $\omega \tau \gg 1$, the electron motion is dominated by the oscillation of the well in the laser field and, consequently, sees a laser-dressed potential well. Because the laser-dressed potential, for $\omega \tau \gg 1$, is different from the undistorted potential, the boundstate eigenvalues change. The regime of $\omega \tau \gg 1$ is, therefore,

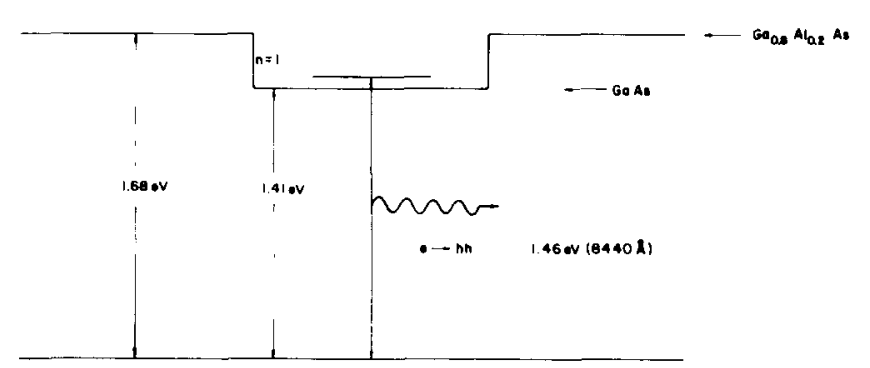

FIG. 1. Energy-band diagram of the GaAs $75-\AA$ quantum well laser of Ref. 5 . The wiggly line indicates the electron-heavy hole $(e-h h)$ transition responsible for the lasing emission at $8440 \AA$. The respective band-gap energies of GaAs quantum well and the $\mathrm{Ga}_{1.8} \mathrm{Al}_{02}$ As barrier are also indicated. 


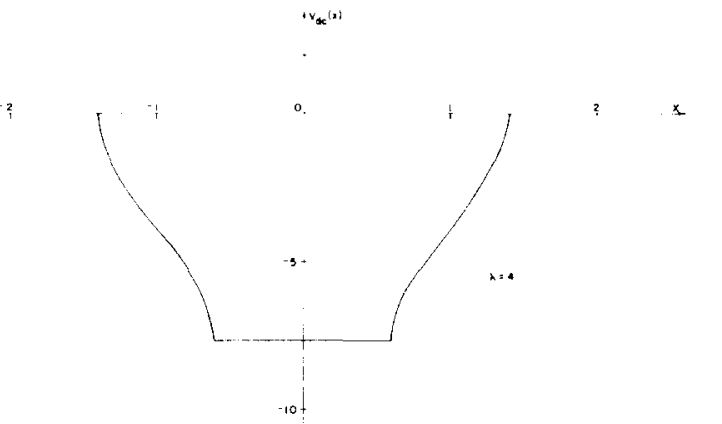

FIG. 2. Dressed dc potential of the $75-\AA$ quantum well of Ref. 5 , as a function of the position, for a dressing laser field strength corresponding to $\lambda=0.4$. The energy is measured in units of $2 \hbar^{2} / m a_{0}^{2}$ and the length in units of $a_{0} / 2$.

the case for which the quantum well laser emission can be tuned by an external laser field.

The solution of Eq. (4) with $\omega \tau \gg 1$ can be calculated by noting that $V[x-\delta(t)]$ is a periodic function in time, for any position $x$. Thus, expanding $V[x-\delta(t)]$ in a Fourier series, one has

$$
V[x-\delta(t)]=\sum_{k} \sum_{v=-\infty}^{+\infty}(-i)^{v} V_{k} J_{v}(k a) e^{i k x} e^{-i v w t},(5)
$$

where $V_{k}$ is the $k$ th Fourier component of $V(x)$ and $J_{v}(z)$ is the Bessel function of order $v$. Since $\omega \tau \gg 1$, we may say that the electron is actually subjected to the time average potential $\langle V(x-\delta(t)\rangle$. Thus, the dominant term in Eq. (5) is the $v=0$ term, hereafter called $V_{\mathrm{dc}}$ :

$$
V_{\mathrm{dc}}=\sum_{k} V_{k} J_{0}(k a) e^{\cdots i k x} .
$$

For a sufficiently deep well, $\tau$ is equal to $2 m a_{0}^{2} / \pi \hbar$. Thus, for a GaAs quantum well laser with $a_{0}=100 \AA$, the regime $\omega \tau \gg 1$ corresponds to $\omega \gg 2 \times 10^{13} \mathrm{~s}^{-1}$, say $\omega>10^{14} \mathrm{~s}^{-1}$. For $\omega>10^{14} \mathrm{~s}^{-1}$, the laser-dressed bound states are then given by the solutions to

$$
\left(-\frac{\hbar^{2}}{2 m} \frac{d^{2}}{d x^{2}}+V_{\mathrm{dc}}\right) \phi=E \phi .
$$

Performing the integration indicated in Eq. (6) one arrives at the following analytical expression for $\boldsymbol{V}_{\mathrm{dc}}$ :

$$
V_{\mathrm{dc}}=-\left(V_{0} / \pi\right)[I(\alpha)-I(\beta)],
$$

where $\alpha=\left(x+a_{0} / 2\right) / a, \beta=\left(x-a_{0} / 2\right) / a$, and the function

TABLE I. Ground-state energy of a GaAs 75-A-wide quantum well for $V_{0}=270 \mathrm{meV}$ and $V_{0}=500 \mathrm{meV}$, as a function of the laser electric field strength $(\lambda)$. The energies are measured in units of $2 \hbar^{2} / m a_{0}^{2}=34.92 \mathrm{meV}$, and the lengths in units of $a_{0} / 2$. The parameter $\lambda$ is related to the dressing laser frequency and intensity by $I=0.89 \times 10^{-57} \omega^{4} \lambda^{2}\left(\mathrm{GW} / \mathrm{cm}^{2}\right)$.

\begin{tabular}{lcc}
\hline \hline$\lambda$ & $\begin{array}{c}E_{1} \\
\left(V_{0}=270 \mathrm{meV}\right)\end{array}$ & $\begin{array}{c}E_{1} \\
\left(V_{0}=500 \mathrm{meV}\right)\end{array}$ \\
\hline 0 & 6.38 & 12.75 \\
0.4 & 6.26 & 12.48 \\
0.6 & 5.95 & 11.94 \\
0.8 & 5.51 & 11.12 \\
0.9 & 5.25 & 10.58 \\
1.3 & 3.91 & 7.66 \\
1.4 & 3.70 & 7.17 \\
\hline \hline
\end{tabular}

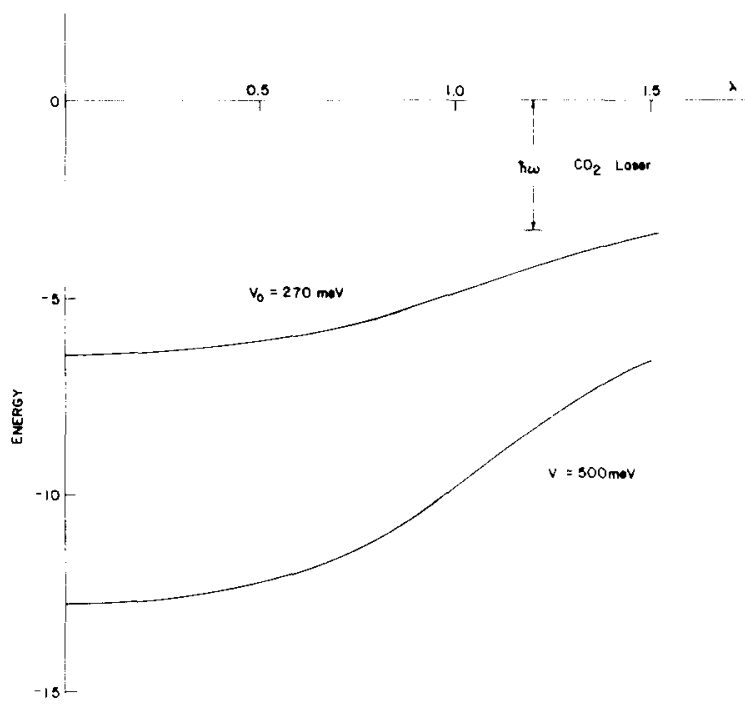

FIG. 3. Ground-state energy of an electron in a laser-dressed 75 -A quantum well as a function of the dressing laser field strength for two different well depths $V_{0}$. The vertical arrow indicates the energy (in units of $2 \hbar^{2} / m a_{0}^{2}$ $=34.92 \mathrm{meV}$ ) of a $\mathrm{CO}_{2}$ laser photon

$I(z)$ is defined by ${ }^{7}$

$$
I(z)= \begin{cases}\pi / 2 & z>1, \\ \arcsin (z) & z^{2}<1, \\ -\pi / 2 & z<-1 .\end{cases}
$$

In Fig. 2 we show the laser-dressed dc potential for a $75-\AA$ quantum well $270 \mathrm{meV}$ deep, for a typical driving laser electric field stength. In our calculations we measured the energies in units of $2 \hbar^{2} / m a^{2}$, and the lengths in units of $a_{0} / 2$. We also introduced the dimensionless parameters $\sigma=m a_{0}^{2} V_{0} /$ $2 \hbar^{2}$, as a measure of the well depth, and $\lambda=2 a / a_{0}$ as a measure of the amplitude $a=e A / m c \omega$ of the electron oscillation in the dressing laser field in units of $a_{0} / 2$. The parameter $\lambda$ is related to the laser intensity $I$ by $I=n \mathrm{~cm}^{2} \omega^{4} a_{0}^{2} \lambda^{2} / 32 \pi e^{2}$, where $n$ is the refractive index. For the case of GaAs ( $\left.m=0.07 m_{0}, n=3.4\right)$, using a low absorption wavelength such as the $10.6 \mu \mathrm{m}$ of the $\mathrm{CO}_{2}$ laser the parameter $\lambda$ (in $\left.\mathrm{GW} / \mathrm{cm}^{2}\right)$ is

$$
I=1.55 \alpha_{0}^{2} \lambda^{2}\left(\mathrm{GW} / \mathrm{cm}^{2}\right),
$$

where $\alpha_{0}$ is the well width in units of $100 \AA$. Thus, for a well $100 \AA$ wide $\left(\alpha_{0}=1\right), \lambda=1$ corresponds to $\mathrm{aCO}_{2}$ intensity of $1.55 \mathrm{GW} / \mathrm{cm}^{2}$. It follows from Fig. 2 that in the presence of a dressing laser the width of the dressed potential increases. This means that the effect of the dressing laser points in the direction of a weakening of the electron binding to the well.

In Table I and Fig. 3, we present the results of our calculation for the ground-state energy, as a function of the dressing laser field, for a 75- $\AA \mathrm{GaAs}$ quantum well with two different well depths. For these values of well parameters the energies are measured in units of $2 \hbar^{2} / m a_{0}^{2}=34.92 \mathrm{meV}$. The energy eigenvalues were obtained by direct numerical integration of Eq. (7). Figure 3 shows us that, on increasing the laser field strength, the ground-state energy decreases. This means that for very large values of $\lambda$ there is a tendency for the energy spectrum to become essentially continuous. 
Here, however, one should exercise some caution since much before one can reach large values of $\lambda$ the electron may be ionized by the dressing laser photon itself. To see this, let us consider the specific case where the driving laser is a $\mathrm{CO}_{2}$ laser. Referring to Fig. 3 we see that on increasing the laser field strength one may eventually reach a point where the ground-state energy equals the laser photon energy, and the approximation $\omega \tau \gg 1$ breaks down for the laser-dressed state. For the case of the GaAs quantum well presently considered and for a $\mathrm{CO}_{2}$ laser as the dressing laser, this happens at $\lambda \cong 1.5$ or for an intensity of $1.96 \mathrm{GW} / \mathrm{cm}^{2}$. At this point the electron gets ionized, thereby ceasing the lasing activity.

To apply these features to obtain tunability of the QWL emission, let us write the lasing frequency $\omega_{L}$ (electronheavy hole transition) for the quantum well of Fig. 1 $\left(V_{0}=270 \mathrm{meV}\right)$ as

$$
\hbar \omega_{L}=1.41+0.27-\left|E_{1}(\lambda)\right| \quad(\text { in } \mathrm{eV}) .
$$

For $\lambda=0,\left|E_{1}(\lambda=0)\right|=0.22 \mathrm{eV}$ and the lasing wavelength turns out to be $8440 \AA$. Now, for $\lambda=0.9$, it follows from Table I that $\left|E_{1}(\lambda=0.9)\right|=0.18 \mathrm{eV}$, so that the lasing wavelength becomes equal to $8215 \AA$. This means that by varying the dressing laser intensity one may, in principle, tune the lasing frequency in the interval between 8440 and $7.700 \AA$. For the 75 -A quantum well with $V_{0}=270 \mathrm{meV}$, assuming a $\mathrm{CO}_{2}$ laser as the dressing laser, $\lambda=0.9$ corresponds, according to Eq. (10), to an intensity of $710 \mathrm{MW} / \mathrm{cm}^{2}$, which for an active area of $8.25 \times 10^{-4} \mathrm{~cm}^{2}$, corresponds to a peak power of $0.60 \mathrm{MW}$. Even though this value for the laser power seems to be relatively modest it may, however, be sufficiently high for thermal effects to smooth out the above predictions. This entails that one should impose another condition, namely that the temperature rise $\Delta T$ induced by the laser beam should be negligible. This additional condition can be easily met by working with nano- or subnanosecond pulses, as follows. The temperature rise $\Delta T$ due to the absorption of a $10.6-\mu \mathrm{m}$ square laser pulse of duration $t_{p}$ is equal to $\Delta T=\alpha I t_{p} / \rho c$, where $\alpha$ is the absorption coefficient, $\rho$ is the density, and $C$ is the specific heat. For GaAs one has ${ }^{8,9} \alpha \cong 9$ $\mathrm{cm}^{-1}$ at $10.6 \mu \mathrm{m}$ for a carrier density of $3 \times 10^{17} \mathrm{~cm}^{-3}$, $c=0.35 \mathrm{~J} / \mathrm{g}{ }^{\circ} \mathrm{C}$ and $\rho=5.32 \mathrm{~g} / \mathrm{cm}^{3}$. Thus, for a pulse duration of $1 \mathrm{~ns}, \Delta T$ equals to $3.4^{\circ} \mathrm{C}$ for an intensity of $0.71 \mathrm{GW} /$ $\mathrm{cm}^{2}$, which is quite acceptable. Note that the pulse energy is only $0.60 \mathrm{~mJ}$.

Finally, it should be remarked that this shifting of the laser emission towards shorter wavelengths due to the quantum well dressing is accomplished at the expense of an increase of the threshold current density $J_{\mathrm{th}}$. The reason for this increase of $J_{\text {th }}$ as a function of $\lambda$ is twofold. First, the quantum well dressing entails an increase of the active region width from $a_{0}$ to $a_{0}(1+\lambda)$. Second, and most important of all, as the laser emission of the dressed well occurs at higher energies, one should expect a decrease of the radiative electron-hole recombination rate. In fact, since the radiative recombination rate $R$ is proportional to the probability of occupation of the well ground state, in the presence of the dressing laser one has $R=R_{0} \exp \left[-\Delta E_{1}(\lambda) / k_{B} T\right]$, where $R_{0}$ is the radiative recombination rate of the laser-free system and $\Delta E_{1}(\lambda)=E_{1}(\lambda)-E_{1}(\lambda=0)$ is the energy shift of the $n=1$ level as a function of $\lambda$. Introducing these changes into the normal expression for the threshold current density as given in Ref. 5, the new threshold current density for the $75-\AA$ quantum well laser $\left(V_{0}=270 \mathrm{meV}\right)$, operating at room temperature and for $\lambda=1\left[\Delta E_{1}(\lambda)=0.05 \mathrm{eV}\right]$, turns out to be $980 \mathrm{~A} / \mathrm{cm}^{2}$ which is roughly three times larger than the laser-free threshold current.

'L. L. Chang, L. Esaki, and R. Tsy, Appl. Phys. Lett. 24, 593 (1974).

${ }^{2}$ R. Dingle, W. Wiegmann, and C. H. Henry. Appl. Phys. Lett. 33, 827 (1974).

N. Holonyak, R. M. Kolbas, R. D. Dupuis, and P. D. Dapkus, IEEE J. Quantum Electron. QE-16, 170 (1980).

${ }^{4}$ W. T. Tsang, Appl. Phys. Lett. 39, 786 (1981).

'D. Kasemset, C. S. Hong, N. B. Patel, and P. D. Dapkus, Appl. Phys. Lett. 41, 912 (1982).

${ }^{4}$ C. A. S. Lima and L. C. M. Miranda, Phys. Rev. A 23, 3335(1981); L. C. M Miranda, Solid State Commun. 45, 783 (1982).

${ }^{7}$ I. S. Gradshteyn and I. M. Ryzhik, Table of Integrals, Series and Products (Academic, New York, 1965), p. 744.

${ }^{\star}$ J. I. Pankove, Optical Properties in Semicoductors (Prentice-Hall, Englewood Cliffs, NJ, 1971), p. 75

'A. S. Grove, Physics and Technology of Semiconductor Devices (Wiley, New York, 1967), p. 102 